\title{
Corela
}

Cognition, représentation, langage

$1-1 \mid 2003$

Vol. $1, \mathrm{n}^{\circ} 1$

\section{La faculté de langage : travaux récents d'inspiration fonctionaliste sur son architecture, ses universaux, son émergence et sa transmission}

Jacques François

\section{(2) OpenEdition}

\section{Journals}

Édition électronique

URL : http://journals.openedition.org/corela/641

DOI : $10.4000 /$ corela.641

ISSN : 1638-573X

Éditeur

Cercle linguistique du Centre et de l'Ouest - CerLICO

\section{Référence électronique}

Jacques François, «La faculté de langage : travaux récents d'inspiration fonctionaliste sur son architecture, ses universaux, son émergence et sa transmission », Corela [En ligne], 1-1 | 2003, mis en ligne le 01 avril 2003, consulté le 19 avril 2019. URL : http://journals.openedition.org/corela/641 ; DOI : 10.4000/corela.641

Ce document a été généré automatiquement le 19 avril 2019.

\section{(c) (i) (3)}

Corela - cognition, représentation, langage est mis à disposition selon les termes de la licence Creative Commons Attribution - Pas d'Utilisation Commerciale - Partage dans les Mêmes Conditions 4.0 International. 


\title{
La faculté de langage : travaux
} récents d'inspiration fonctionaliste sur son architecture, ses universaux, son émergence et sa transmission

\author{
Jacques François
}

\begin{abstract}
Il ne s'agit pas de considérer le langage comme l'a
formulé un jour Saussure uniquement en soi et pour soi, mais au regard de la totalité qui construit la pensée et l'action de l'homme et peut-être même au-delà dans ce qui nous entoure dans la nature

[Hansjakob Seiler $]^{1}$
\end{abstract}

1 Cet article reprend le contenu de ma conférence du 6 décembre 2001 dans le cadre du DEA de Sciences du Langage et du CRISCO (Université de Caen). Cette conférence constitue un jalon dans une série de travaux consacrés à la diversité des linguistiques fonctionnelles dans la dernière décennie du XX $\mathrm{XX}^{\mathrm{e}}$ siècle $^{2}$.

Dans l'introduction (François 1998) au numéro de la revue VERBUM intitulé Orientations récentes en grammaire fonctionnelle, entre sciences cognitives et biologie (1998/3), j'ai abordé en première partie le classement des théories grammaticales (centrées ou pas sur la syntaxe) selon van Valin \& LaPolla (1997, Chapitre 1), le fonctionalisme sémiotique de la Grammaire Cognitive qui conçoit la syntaxe comme la composition hiérarchisée d'assemblées symboliques (Langacker 1995), la théorie de la compétition entre motivations externes et internes (DuBois 1985), les deux débats distincts sur l'autonomie de la syntaxe ou de la grammaire (Croft 1995) et la place respective des processus guidés par la grammaire vs. par le savoir dans la compréhension de texte selon Kintsch 1995 et Givón 1995b. Dans la seconde partie consacrée aux nouveaux modèles cognitifs et biologiques de l'émergence 
du langage articulé et du changement linguistique, j'ai comparé les points de vue défendus dans ce numéro de VERBUM

- par T. Givón (1998) sur les bases neurobiologiques de l'émergence du langage articulé,

- par W.Croft (1998) sur l'applicabilité d'un modèle épistémologique biologique pour la typologie fonctionnelle des langues et sur le changement linguistique envisagé dans un paradigme évolutionniste (Croft 1996) ${ }^{3}$,

- et par S. Kirby $(1998,1999)$ sur la possibilité de simuler la diffusion typologique d'une structure grammaticale à l'aide d'un modèle de compétition. L'option adaptative illustrée par ces différents travaux permet de comprendre pourquoi le poids des deux principes fondamentaux d'iconicité et d'économie décroît au cours de l'évolution des langues.

Mon propos est ici de mettre en valeur des travaux d'origine disciplinaire très variée parus entre autres dans quatre ouvrages collectifs de référence. Le premier ouvrage par ordre chronologique, sous la responsabilité de Jeffrey Elman et al. (1996), discute l'innéité des facultés à partir des moyens de simulation connexionniste, le second, coordonné par M. Tomasello (1998), met en évidence les traits communs aux approches fonctionnelle et cognitive en sciences du langage, le troisième, coordonné par M. Darnell et al. (1999) ouvre en deux remarquables volumes ${ }^{4}$ la controverse entre les points de vue formaliste et fonctionaliste en épistémologie de la linguistique, tandis que le quatrième, sous la responsabilité de B. MacWhinney (1999), fait le point sur l'émergence du langage (essentiellement d'un point de vue développemental).

Dans la première section, je confronterai plusieurs points de vue sur la controverse épistémologique entre fonctionalisme et formalisme en linguistique. Dans la seconde j'aborderai la question du type de corrélation entre les universaux cognitifs et les universaux du langage à travers la seule théorie de la grammaticalisation de schèmes cognitifs proposée par B. Heine ${ }^{5}$. La troisième section est consacrée à un aperçu de la théorie de l'anthropologue Terrence Deacon sur l'acquisition de traits comportementaux qui entrent dans le patrimoine génétique (conception défendue à l'origne par J.M. Baldwin), sur le caractère définitoire de la faculté de langage dans l'émergence de l'espèce humaine et sur la notion d'homo symbolicus qui en découle. La section 4 porte sur les propriétés du langage susceptible d'être innées. Après avoir rappelé une série d'arguments proposés par Elman et al. (1996), j'essaie de mettre de l'ordre dans une discussion animée sur le 'paradoxe' de l'acquisition des structures linguistiques qui s'est dans une large mesure poursuivie à travers les numéros de la revue Cognitive Linguistics en réaction à la thèse de Ray Jackendoff $(1996,1997)$. Enfin la section 5 présente deux tentatives de simulation de l'ontogénèse du langage et de la transmission des langues par réseaux neuronaux ${ }^{6}$.

Seule la section 1 a un caractère contradictoire et donne la parole aux tenants de grammaires 'formelles' et 'fonctionnelles'. Mais le débat est mal posé en ces termes, car la Functional Grammar de Dik (1997) présente un formalisme sémantique avancé et la Role and Reference Grammar (van Valin \& LaPolla 1997) intègre une syntaxe et une sémantique formelles. Concernant la faculté de langage, le débat majeur porte sur l'autonomie de la syntaxe par rapport à la sémantique et plus largement sur l'autonomie de la grammaire (morphologie, syntaxe et sémantique confondues) par rapport aux motivations externes (d'ordre cognitif, culturel et social), comme l'a remarquablement exposé W. Croft (1995). Dans les sections 3 à 5 , je me contente de rassembler des arguments provenant de chercheurs en anthropologie (Deacon), en psychologie cognitive (Braine, Tomasello), en intelligence artificielle (Elman) ou polyvalents (Kirby) propres à modéliser l'acquisition 
de la faculté de langage par l'individu (de quel ordre sont les 'principes' et les 'paramètres' ?) et par l'espèce, (comment les 'principes' ont-ils acquis ce statut ?).

\section{Sur la controverse épistémologique entre fonctionalisme et formalisme en linguistique}

6 Dans son introduction au volume collectif, The new psychology of language (1998), M. Tomasello déclare:

"If we are ever to understand the many complexities of language, what we need are not more elegant mathematical formalizations, but rather more cooperation between psychologists and linguists in helping to determine how basic cognitive and social processes operate in the specialized domain of human linguistic communication." (p.XXI)

7 Cette position rappelle d'une part les conclusions positives de Kintsch (1995) et Givón (1995) sur la pertinence de la collaboration entre linguistes et psychologues expérimentalistes dans le domaine de la compréhension de texte, mais constitue surtout un rejet des modèles formalistes ou plus exactement strictement computationnels ${ }^{7}$. Il est instructif de laisser la parole à des protagonistes du débat plus ou moins engagés dans le "camp" formaliste ou fonctionaliste pour saisir les enjeux de la controverse.

\subsection{Critique des bases du fonctionalisme}

8 Anderson (1999) illustre un point de vue formaliste strict. Il reproche aux fonctionalistes de ne pas se contenter de formuler l'hypothèse d'une perméabilité des structures syntaxiques vis-à-vis de facteurs de signification et d'usage, mais de concevoir la mise en évidence de cette perméabilité comme une stratégie de recherche (p.120). Selon Anderson, la méthode des "formalistes" consiste à formuler l'hypothèse du fonctionnement autonome de composantes distinctes du savoir linguistique et de la descriptibilité des phénomènes complexes par leur interaction. L'étude de la capacité générative du langage humain (objectif déclaré des formalistes) invalide les données sur l'usage et la fréquence "et même la question de savoir si une possibilité donnée est effectivement instanciée dans une quelconque langue" (p.120-1). Le domaine de recherche des formalistes est ce que le language est (la compétence), tandis que le domaine qu'il concède aux fonctionalistes est celui de ce que le langage fait (la performance), qu'il caractérise comme la "prise en compte unifiée de l'activité des utilisateurs du langage humain". Anderson entend fixer une hiérarchie des priorités :

- les grammaires (internes) de la compétence (ou I-grammars, relevant de modèles formels) sont le noyau dur fondé sur la distinction entre principes et paramètres. Elles seraient seules aptes à rendre compte de l'aptitude humaine à manier les langues comme un outil abstrait déconnecté des besoins de communication ;

- les grammaires (externes) de la performance (ou E-grammars, relevant de modèles fonctionnels) rendraient compte de l'usage effectif des langues dans la communication, ce qui selon Anderson constitue une problématique extérieure à la grammaire au sens étroit.

La contribution de D. Nettle dans le même volume (1999) vise à pointer les difficultés du fonctionalisme en biologie, qui se répercutent sur son emploi en linguistique. Tout comme Givón (1995), dont le fonctionalisme est pourtant indiscutable, Nettle critique le dogme fonctionaliste de la structuration adaptative (emprunté à la théorie de l'évolution 
en biologie). Il présente comme circulaire et empiriquement improductive l'hypothèse de l'adaptativité linguistique - c'est-à-dire d'une relation fonctionnelle entre le degré d'utilité communicationnelle ou cognitive de formes linguistiques et la probabilité de leur adoption. Il commente ainsi l'objection de Lass (1980) selon laquelle "le problème avec l'argument fonctionnel c'est que vous ne pouvez pas perdre" :

"On the one hand, it is assumed that the linguistic system after the development in question is better adapted than the one prior to it, and a post hoc reason for this is found. On the other, the development is said to have been caused by this functional pressure" (Nettle 1999: 450)

En cela, Nettle est implicitement en accord avec la critique virulente de T. Givón dans l'introduction de Functionalism and Grammar (1995, p.xvi) contre le réductionnisme fonctionaliste caractérisé par la thèse de l'adaptation triomphante.

\subsection{La prise en compte des motivations fonctionnelles dans un cadre génératif (F. Newmeyer 1999)}

11 Plus enrichissante est l'analyse de Frederick Newmeyer (d'orientation explicitement générativiste) qui, dans le prolongement de sa remarquable synthèse, Language form and language function (1998) commence par offrir une réanalyse fonctionnelle du phénomène de 'preposition stranding' caractéristique de l'anglais (cf. 1-5) :

(1) Who did you speak to?

(2) Mary was spoken to.

(3) Who did you read a book about?

(4) Which shoes did you walk from Seattle to Tacoma in?

(5) Which knife shall we use to cut the turkey with?

12 Les générativistes expliquent le "preposition stranding" à partir d'une réanalyse, le syntagme prépositionnel [to who] étant dissocié de telle sorte que la préposition to devient l'équivalent d'une particule verbale $[$ talk + to]. Cette restructuration permet le déplacement autonome de who en tête de phrase interrogative :

You talked ${ }_{\mathrm{pp}}$ [to who]

$>$ You $_{\mathrm{v}}$ [talked to] who

$>$ Who $_{\mathrm{i}}$ did you [talk to] $\mathrm{e}_{i}$ ?

13 Cependant Newmeyer fait valoir que la réanalyse suppose un lien étroit entre le SPrep et le verbe, c'est-à-dire un statut d'objet (cf. 1-2) et non d'adjoint. Cependant les exemples (3-5) illustrent le déplacement d'un constituant de SPrep à statut d'adjoint. Observant que dans chacune de ces phrases l'objet de la préposition peut être interprété comme un focus d'attention, Newmeyer argumente contre le recours à un mécanisme transformationnel ad hoc, et préfère recourir à un principe fonctionnel : un constituant peut devenir le focus (syntaxique) d'une question s'il peut - avec le syntagme qui le contient - être construit comme un focus (pragmatique) d'attention raisonnable. En conséquence, dans une situation d'énonciation où la destruction de livres peut être construite comme une activité normale, la phrase (6) très étrange peut recevoir une forme enrichie acceptable (cf. 6a / 6b) :

(6)? Who did you destroy a book about?

(6a) Which former party official did the Red Guard destroy more books about: Lin Piao or Liu Shao-Chi?

(6b) The party official that I would really like to destroy a book about is Chao EnLai. 
14 Newmeyer fait donc un grand pas en direction du fonctionalisme "ordinaire" selon lequel les structures sont corrélées individuellement aux motivations fonctionnelles. Cependant il reste un adepte de l'autonomie de la syntaxe, ce qui le conduit à proposer un modèle formaliste dans lequel les structures sont corrélées à un "système structural" lui-même corrélé aux motivations fonctionnelles. Il s'agit d'une "théorie 'pré-contrainte' [ constrained] des moyens par lesquels les pressions fonctionnelles peuvent affecter les grammaires". Son argument majeur (p. 478) est que le jeu de motivations opposées suppose un arbitre qui doit avoir un statut autonome :

"Le système structural autonome est une réponse fonctionnelle naturelle aux exigences externes concurrentes sur la grammaire. La syntaxe autonome est une solution raisonnable au problème posé par le fait qu'il est impossible pour les grammaires de refléter directement une motivation fonctionnelle particulière, puisque de telles motivations tendent à s'opposer les unes aux autres. La syntaxe autonome - encore une fois, un système médiateur entre forme et fonction - sert de 'standard's naturel pour coordonner les deux".

\subsection{L'intégration des deux points de vue illustrée dans la Role and Reference Grammar}

La Grammaire des Rôles et de la Référence que résume van Valin (1996, 2001 : 205-218) et dont van Valin \& LaPolla (1997) fournit une présentation exhaustive, réalise l'intégration des deux perspectives, formaliste (dérivée de la 'Grammaire de Montague' par l'intermédiaire de Dowty 1979) et fonctionaliste, en prévoyant pour la "projection des constituants" des "pièces" (templates) syntaxiques qui ne correspondent pas obligatoirement à des syntagmes complets (il peut s'agir de syntagmes tronqués par exemple par absence d'un membre) qui constituent l'inventaire syntaxique de cette projection.

La sélection et la composition des pièces est pilotée par la structure logique, qui traduit le contenu de l'énoncé dans une notation formalisée, combinée à la projection pragmatique, laquelle fixe le type de topique et de focus représenté dans l'énoncé. A certains arguments de la structure logique peuvent être attachés les macrorôles 'Actor' et ' Undergoer' selon des règles de sémantique lexicale, par exemple en raison de l'identification d'un référent contrôlant le procès ou directement affecté par celui-ci, et de sémantique grammaticale, par exemple à la suite d'un "réarrangement des actants" (S. Dik) c'est-à-dire d'une diathèse passive, récessive ou causative.

17 Une troisième projection, dite projection des opérateurs gère la réalisation morphosyntaxique des catégories fonctionnelles, prédicatives pour le noyau prédicatif ou "core": temps, aspect, modalité, force illocutoire, et référentielles pour le syntagme nominal référentiel : détermination, nombre et quantification, qualification adjectivale.

Un avantage majeur de cette théorie est l'absence de toute transformation: par corrélation entre les différentes composantes mentionnées ci-dessus, les constituants sont disposés directement à leur place définitive. Ainsi, pour la phrase Who did you speak to ?, la projection des constituants ne comporte pas de trace ni de constituant déplacé : le SPrep [to Ø] est réduit à sa tête et le pronom interrogatif est directement disposé dans une position intitulée "pre-core slot" destinée à accueillir des constituants "échoués" (cf. preposition stranding!). L'affectation de who à cette position est pilotée par la focalisation interrogative sur le destinataire de l'acte de parole, qui est identifiée dans la projection pragmatique. En d'autres termes, le locuteur décide de formuler une interrogation 
focalisée sur le destinataire de l'acte de parole. Cette intention de communication est notée dans la projection pragmatique, laquelle gère la sélection de la pièce "proposition avec pre-core slot" $\left(\left[[w h o]_{\text {pre-core slot }}[\ldots]_{\text {Core }}\right]_{\text {prop }}\right.$ ), la dite proposition dominant un noyau prédicatif (core) tronqué $\left.\left[[. . .]_{\mathrm{SN}}[\text { speak- }]_{\text {Pred }}[\text { to }]_{\text {Sprep }}\right]\right]_{\text {Core. }}$.

\subsection{Une illustration de l'intrication entre la syntaxe et la sémantique : les grammaires de construction}

Dans sa contribution à l'ouvrage collectif coordonné par M. Tomasello (ed., 1998), W. Croft (1998: 90-91) défend un point de vue radical sur la liberté d'insertion des unités lexicales dans des patrons distributionnels :

"Jusqu'à un certain point, tout mot peut être employé en principe dans n'importe quelle construction. De ce fait les patrons distributionnels n'établissent pas de catégories grammaticales au sens strict. Ce qui compte, c'est l'interprétation sémantique d'un mot dans une construction particulière (..) cette interaction entre les constructions grammaticales et les mots que les locuteurs y insèrent est la source de la richesse et de la flexibilité du langage comme moyen de communiquer le vécu. La flexibilité de la grammaire est limitée à un certain point par nos attentes sur ce à quoi ressemble le monde et sur la manière dont différents types d'événements peuvent être construits mentalement de manière plausible".

Cette conception est apparentée à celle du psychologue Martin Braine (1992) sur l'acquisition primaire des structures sémantiques et secondaire des structures syntaxiques propres à les exprimer ( $c f$. plus bas \$4.2), mais elle est surtout en accord avec les "grammaires de construction" développées par C. Fillmore, P. Kay et A. Goldberg.

21 Les ouvrages qui nous servent de référence comportent deux articles d'A. Goldberg (1998, 1999). Ils ont un même objet, la capacité de certains patrons distributionnels à véhiculer en priorité certaines configurations de participants, l'article de 1999 étant plus particulièrement consacré à l'acquisition des potentialités de ces "constructions". L'auteur illustre son propos à partir de deux types de phrases élémentaires. Pour le type 1 , l'interprétation semble fondée sur la projection du verbe sur sa structure argumentale ; pour le type 2, l'interprétation suppose une autonomie sémantique de la construction ${ }^{10}$. Le tableau 1 montre comment un verbe sémantiquement peu spécifié (go, do, give, make, put $)^{11}$ peut être remplacé dans une construction par un verbe très spécifique, par exemple do > eyebrow / [ $\left.\mathrm{N}_{-} \mathrm{N}\right]$.

Tableau 1 : La substitution d'un verbe hautement spécifique à un 'light verb' dans des structures argumentales de l'anglais

\begin{tabular}{|l|l|l|}
\hline $\begin{array}{l}\text { structure } \\
\text { argumentale }\end{array}$ & type 1 & type 2 \\
\hline N V PartDirect N & Pat went down the street & the truck rumbled down the street \\
\hline N V N & Pat did her homework & Pat eyebrow'd her surprise* \\
\hline N V N N & Pat gave Chris a cake & We will overnight you that package** \\
\hline N V N Adj & Pat made Chris happy & He kissed mother unconscious \\
\hline
\end{tabular}




\begin{tabular}{|l|l|l|}
\hline N V N PrepLoc N & $\begin{array}{l}\text { Pat put the book on the } \\
\text { table }\end{array}$ & $\begin{array}{l}\text { They couldn't manage to pray the two little girls } \\
\text { home again*** }\end{array}$
\end{tabular}

*Pat marqua sa surprise d'un froncement de sourcils

** Nous vous transmettrons ce paquet du jour au lendemain

*** Ils n'ont pas réussi à ramener les deux petites filles à la maison par leurs prières.

Le tableau 2 réinterprète ce phénomène à partir de la corrélation entre une structure sémantique, une structure syntaxique et un choix de verbes propres à entrer dans ces structures. Pour exprimer l'entrée d'une mouche dans une pièce $\left(\mathrm{X}_{f l y}\right.$ moves to $\left.\mathrm{Y}_{\text {room }}\right)$, le verbe buz indiquant le bruit associé au déplacement de la mouche est substituable au verbe ordinaire fly. Et pour exprimer le découpage d'une pièce de viande, cube peut se couler dans la structure argumentale de slice ou cut et spécifier ainsi la forme des morceaux découpés ${ }^{12}$.

Tableau 2 : Le statut syntactico-sémantique des "constructions" de l'anglais (Obl : oblique)

\begin{tabular}{|l|l|l|}
\hline Construction / Exemple & Signification & Structure \\
\hline $\begin{array}{l}\text { 1. Intransitive motion } \\
\text { The fly buzzed into the room. }\end{array}$ & X moves to Y & Subj V Obl \\
\hline $\begin{array}{l}\text { 2. Transitive } \\
\text { Pat cubed the meat. }\end{array}$ & X acts on Y & Subj V Obj \\
\hline $\begin{array}{l}\text { 3. Resultative } \\
\text { She kissed him unconscious. }\end{array}$ & X causes Y to become Z & Subj V Obj CoMP \\
\hline $\begin{array}{l}\text { 4. Double object } \\
\text { Pat faxed Bill the letter. }\end{array}$ & X causes Y to receive Z & Subj V Obj Obj2 \\
\hline $\begin{array}{l}\text { 5. Caused-motion } \\
\text { Pat sneezed the foam off the cappucino. }\end{array}$ & X causes Y to move Z & Subj V Obj Obl \\
\hline
\end{tabular}

L'article de 1999 tire les conséquences de cette souplesse pour l'acquisition de l'anglais comme langue maternelle. Alors que Hopper (1987) regardait la grammaire comme émergeant continuellement durant la production du discours, Goldberg défend la position que la grammaire émerge primairement durant l'acquisition initiale à partir de la combinaison d'un input linguistique, des exigences fonctionnelles de la communication et d'aptitudes et contraintes cognitives. Une fois que la grammaire est acquise, l'auteur admet qu'elle a un statut hautement conventionnalisé et que, même si des changements minimes se produisent constamment dans le système, celui-ci est globalement stable. Cette position n'est donc finalement pas très éloignée de celle de Newmeyer (\$1.2) pour qui le système structural autonome arbitre au mieux la compétition des motivations fonctionnelles une fois que l'apprenant a repéré son espace de liberté et les contraintes imposées pour un bon déroulement de la communication. 


\section{Sur les universaux et la typologie des langues : la grammaticalisation diversifiée des schèmes cognitifs selon Bernd Heine}

Pour B. Heine, célèbre africaniste et typologue, il existe une corrélation relativement directe entre les universaux de cognition et les universaux du langage par l'intermédiaire de la grammaticalisation de schèmes cognitifs :

"the question of why language is structured the way it is can be answered meaningfully only if one takes the cognitive foundations into consideration from which language structure is derived" (B. Heine 1997c: 21)

alors que C. Hagège (1998: 46) défend la thèse inverse de la variabilité interculturelle des visions du monde, entre autres d'ordre spatial, en fonction de modèles sociaux et culturels variés, ce qui ébranle l'hypothèse que le substrat cognitif commun suffit à produire des homologies entre langues. Heine reconnaît certes l'argument de la variété :

"human conceptualization, like the way it shapes language structure, is far from uniform across cultures; rather, it may offer various solutions to the problems it is meant to solve" (1997b: 14)

mais il y oppose dans la même phrase l'argument du vivier restreint des conceptualisations :

"At the same time, however, both the number and the kind of solutions developed to cope with a given problem are limited. What it means is that there is both diversity and unity: The human species, irrespective of whether it is located in Siberia or the Kalahari Desert, has essentially the same pool of options for conceptualization".

Dans son ouvrage consacré à l'origine des divers moyens d'expression de la relation de possession (1997a), Heine identifie d'un côté neuf schèmes cognitifs d'événements d'où dérivent tous les moyens d'exprimer la possession par prédication ou par détermination, et de l'autre trois variantes cibles : avoir : $\mathrm{X}$ a/possède $\mathrm{Y}$, appartenir : $\mathrm{Y}$ appartient à $\mathrm{X}$, déterminatif : le $\mathrm{Y}$ de $\mathrm{X}$ ( $c f$. Tableau 3). Le transfert cognitif entraîne un processus de grammaticalisation qui généralement conduit à un changement de statut catégoriel de l'expression source.

Tableau 3 : Les principaux schèmes évenementiels source et les constructions possessives qui en dérivent

AVoir : $X$ a/possède $Y$, APPARTENIR : $Y$ appartient à $X$, DÉterminatiF : le $Y$ de $X$

\begin{tabular}{|l|l|l|l|l|}
\hline Formule & $\begin{array}{l}\text { Intitulé du schème } \\
\text { évenementiel }\end{array}$ & avoir & $\begin{array}{l}\text { appar- } \\
\text { tenir }\end{array}$ & déterminatif \\
\hline (1) X prend Y & Action & $\cdot$ & $\cdot$ & \\
\hline (2) Yest situé à Y & Localisation & $\cdot$ & &. \\
\hline (3) X est avec Y & Accompagnement & $\cdot$ & &. \\
\hline (5) le X de Y existe & Génitif & $\cdot$ & & \\
\hline (6) Y existe pour X & Destination & $\cdot$ & $\cdot$ &. \\
\hline
\end{tabular}




\begin{tabular}{|c|c|c|c|}
\hline (8) Y existe à partir de $\mathrm{X}$ & Source & & 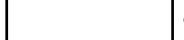 \\
\hline (7) Quant à X, il y a Y & Topique & - & 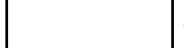 \\
\hline $\begin{array}{l}\text { (9) Y est (une propriété) de } \\
X\end{array}$ & Équation & & l. \\
\hline
\end{tabular}

Dans une même langue, plusieurs schèmes prévalents peuvent coexister. Un argument en faveur de l'universalité des mécanismes qui prennent appui sur les schèmes cognitifs est que les mêmes schèmes dérivent d'autres types de morphèmes, par exemple les classes d'auxiliaires (cf. Heine 1997b).

\section{Un point de vue d'anthropologue sur la place de la faculté de symbolisation et de langage dans l'émergence de l'espèce humaine}

27 Je m'écarterai ici des ouvrages répertoriés jusqu'à présent pour évoquer le point de vue d'un spécialiste d'anthropologie cognitive, Terrence Deacon, de l'Université de Boston, car il corrobore ce qu'a écrit T. Givón (1995a: chapitre IX, 1998a) ${ }^{13}$. Deacon (1997, Chapitre XI : And the word became flesh) défend un modèle d'évolution "baldwinien" (cf. Baldwin 1902 discuté dans Richards 1987). Selon Baldwin, disciple de Darwin, l'apprentissage et la flexibilité comportementale peuvent jouer un rôle (dans l'évolution) en amplifiant et altérant la sélection naturelle, parce que ces aptitudes permettent à des individus de modifier le contexte de sélection naturelle qui affecte leur descendance (Deacon 1997 : 322). La flexibilité comportementale permet à des organismes de s'installer dans ces niches écologiques qui diffèrent de celles de leurs ancêtres avec pour conséquence que les générations postérieures vont être confrontées à un nouvel ensemble de pressions sélectives.

La théorie de Baldwin explique comme les comportements peuvent affecter l'évolution, mais sans avoir à affirmer (contrairement à Lamarck) que les réponses aux exigences environnementales acquises au cours de la vie d'un individu peuvent être transmises directement à sa descendance. Deacon illustre cette thèse à partir de l'évolution de la tolérance au lactose :

"les populations humaines présentant le taux le plus haut d'adultes tolérant le lactose sont celles où des animaux ont été élevées depuis le plus long temps, et celles avec le taux le plus bas sont celles où l'élevage a été introduit le plus récemment ou pas du tout (...) l'emploi de lait animal comme source de nourriture, malgré des difficulés à le digérer pour certains, a favorisé la reproduction de ceux qui le toléraient" (1997 : 323)

Par conséquent, c'est le fait que des comportements appris puissent être hérités qui est à la source du changement évolutionnaire. De la sorte un organisme accède à un répertoire d'adaptations potentielles. Cela conduit Deacon $(1997: 328)$ à prendre position contre la conception innéiste de la grammaire universelle défendue par Pinker (1994): selon l'anthropologue, le cerveau et les aptitudes sensorimotrices des humains présentent bien comme l'affirme Pinker des adaptations à la faculté de langage qui réunies pourraient 
être qualifiées d'"instinct", mais le savoir grammatical en est exclu pour la raison suivante :

"Les attributs les plus universels de structure linguistique sont par nature les plus variables dans la représentation superficielle, corrélés variablement à des tâches de traitement et difficilement localisables dans le cerveau entre individus ou même chez un même individu. De ce fait ils sont les traits du langage qui ont le moins de chances d'avoir développé des supports neuronaux spécifiques. Ces aspects du langage que de nombreux linguistes seraient le plus tentés de ranger dans une Grammaire Universelle sont précisément ceux qui sont inéligibles pour participer à une évolution [à base comportementale] de type Baldwinien." (p. 333).

Deacon considère que l'universalité n'est pas en elle-même un indicateur fiable de ce que l'évolution a bâti dans les cerveaux humains. Il conclut des données typologiques qu'il manque à la logique profonde des règles grammaticales les caractéristiques invariantes qui pourraient leur permettre d'être soumises à une sélection naturelle ${ }^{14}$. Il finit par proposer homo symbolicus (c'est-à-dire une caractérisation d'anthropologie cognitive et non de génétique) comme désignation d'un stade d'évolution des homonidés: tous les hominidés dotés d'une faculté de symbolisation sont effectivement liés par un vivier commun d'informations symboliques, qui est aussi inaccessible aux autres espèces que le sont les gènes humains (p. 341). La propriété d'humanité tiendrait plus à cette aptitude partagée à manier des symboles (transmise par évolution Baldwinienne) qu'au partage de caractéristiques physiques. La caractérisation d'homo symbolicus comme une 'espèce noologique' (nöo-species) distincte d'une désignation biologique d'espèce animale (zöospecies) n'est pas sans rappeler le concept de noosphère comme espace créé par l'émergence de l'humain chez Teilhard de Chardin.

\section{Les modalités de la transmission de la faculté de langage entre générations}

\subsection{Critique générale du domaine d'incidence des caractères innés}

Elman et al. (1996) consacrent 20 pages de leur ouvrage sur l'innéité à présenter 12 arguments sur les représentations innées, avec une référence particulière au langage (p. 371-390). Il n'est pas possible d'évoquer tous ces arguments. Je me contenterai d'évoquer ceux qui sont propres à éclairer la discussion du §4.2.

Un argument souvent mis en avant est celui des pathologies linguistiques sélectives ( selective language impairment : SLI) à base génétique qui a conduit à parler d'un "gène dédié à la grammaire". Une étude consacrée au cas le plus célèbre, une famille anglaise dont de nombreux membres présentent une déficience dans le maniement des morphèmes flexionnels, a montré que la déficience génétique qui touche cette famille est en fait une apraxie orale-faciale, c'est-à-dire une incapacité à articuler correctement les sons et non une déficience d'ordre proprement grammatical. Une autre étude sur des enfants atteints de SLI montre qu'ils souffrent d'un déficit dans le traitement de séquenes temporelles rapides de stimuli auditifs et éventuellement visuels. Là aussi la déficience touche l'ensemble du comportement cognitif et non la simple grammaire.

En aphasiologie, la documentation abondante sur l'aphasie agrammatique de Broca montre que le déficit à la réception ne peut être détecté que si le patient est empêché de recourir à des informations sémantiques pour interpréter les phrases. Ainsi les patients 
traitent en général correctement les phrases passives non réversibles en raison de la sous-catégorisation des actants (ex. L'arbre a été abattu par la foudre) et sont incapables de traiter les phrases réversibles (ex. Le garçon a été poursuivi par la fille). En outre, comme le rappellent Allen \& Seidenberg (1999: 123), Linebarger et al. (1983) ont fourni des preuves à l'encontre de l'affirmation que l'agrammatisme représente une perte sélective de capacité syntaxique, car les patients qui ont eu un résultat non significatif (chance level) à leurs tests de compréhension ont obtenu des résultats élevés dans la tâche de jugement de la grammaticalité de types de phrases similaires. Elman et al. (1996: 321) ajoutent que l'activité linguistique d'évaluation de la grammaticalité met en jeu l'hémisphère droit (non touché par la lésion). Il semble donc que le savoir grammatical ne soit pas dissociable du savoir sémantique et qu'il soit largement distribué dans le cerveau de l'adulte.

Sur la question de la modularité de l'esprit (Fodor 1983), les auteurs observent que toute tâche arbitraire peut produire de l'automaticité si elle est pratiquée assez souvent. L'étude des zones cérébrales en jeu dans la résolution de tâches arithmétiques par un calculateur prodige qui vient d'être menée par l'équipe de neuro-imagerie fonctionnelle de l'université de Caen confirme que le calculateur dédie à cette activité des zones qui ne le sont jamais chez les calculateurs ordinaires. Elmann et al (1996: 387) en concluent : " modules are made, not born", et - se fondant sur de nombreux exemples de simulation par réseaux neuronaux d'un apprentissage non linéaire - ils substituent à la représentation d'une modularité innée celle d'une modularisation progressive. Enfin ils soulignent que la propriété bien documentée de l'âge critique au-delà duquel l'engrammation (imprinting) n'est plus possible $-c f$. Hurford 1999 - est loin de concerner uniquement le langage (elle peut porter sur d'autres facultés et à d'autres âges). Il s'agit de contraintes générales sur l'apprentissage dont la simulation (cf. \$5.1) rend compte sous la forme de changements concrets dans la structure du réseau qui interdisent tout retour à l'état d'origine.

Ces arguments ne mettent pas en cause la thèse indiscutable selon laquelle une partie de notre comportement langagier repose sur un fondement inné. La question est de savoir si l'expérimentation, l'observation des pathologies du langage et la simulation des comportements cognitifs par des réseaux neuronaux corroborent ou mettent en doute la thèse d'un module autonome de traitement du langage. Les données rassemblées par Elmann et al. (1996) - que je n'ai exposées que très partiellement - vont clairement dans le sens d'un apprentissage fondé sur un substrat inné de nature cognitive et non strictement linguistique et au cours d'un cheminement "non linéaire" résultant du traitement combiné de données d'origine diverse.

\subsection{Le type de structure syntactico-sémantique concevable comme inné selon Braine, van Valin et Tomasello}

En 1992 le psychologue Martin Braine propose une révision de la théorie de S. Pinker sur l'innéité des catégories syntaxiques. Les trois primitives développementales de cette théorie sont, dans la reformulation de Braine (1992) :

(1) un mécanisme d'apprentissage recourant à un principe intitulé "de vieilles

règles analysent du matériau nouveau" ;

(2) des catégories sémantiques telles que ARGUMENT, PRÉDICAT, incluant des

catégories ontologiques, par exemple OBJET, LIEU, ACTION, ÉVÉNEMENT ;

(3a) des catégories syntaxiques naturelles $\mathrm{N}, \mathrm{SN}, \mathrm{V}, \mathrm{SV}$, etc.

La proposition de Braine consiste à remplacer (3a) par (3b) : 
(3b) une tendance à classer les mots et les syntagmes qui n'ont pas encore été classés par (1), comme référant à des instances des catégories de (2). le début de l'acquisition du langage comme des catégories innées ayant une réalité biologique ou psychologique séparée de celle de leurs membres canoniques", Braine formule la contre-hypothèse que les catégories syntaxiques émergent sous l'effet de la combinaison des opérations (1) et (3b). Ce faisant, il pose une question cruciale à propos de la fonctionnalité du langage: "Si les enfants commencent par des catégories sémantiques, pourquoi les langues ont-elles des catégories syntaxiques dont l'extension ne correspond pas à celle des catégories sémantiques ?". Sa réponse prudente évoque plusieurs facteurs critiques de disparité entre la catégorisation sémantique et syntaxique :

(1) les limites floues des catégories sémantiques plausibles de la langue de l'esprit;

(2) la superposition et la compétition entre catégories sémantiques (par exemple les rôles sémantiques) et catégories pragmatiques (par exemple les fonctions de topique et de focus) qui doivent être corrélées avec des structures syntaxiques ;

(3) la sensibilité des apprenants aux similarités phonologiques entre les mots ;

(4) et le nombre limité des positions structuralement distinctives en raison de la linéarité consubstantielle au langage.

Van Valin \& LaPolla (1997) exploitent largement l'argumentaton de Braine (1992) dans leur Epilogue "The goals of linguistics revisited". Ils illustrent l'application de la primitive (3b) de Braine par deux arbres représentant la transition d'une analyse en termes de catégories ontologiques vers l'analyse syntaxique dans le format de la Role and Reference Grammar (cf. figure 1).

Figure 1. De lanalyse en termes de catégories ontologiques à l'analyse en catégories syntaxiques (van Valin \& LaPolla $1997: 643$ )

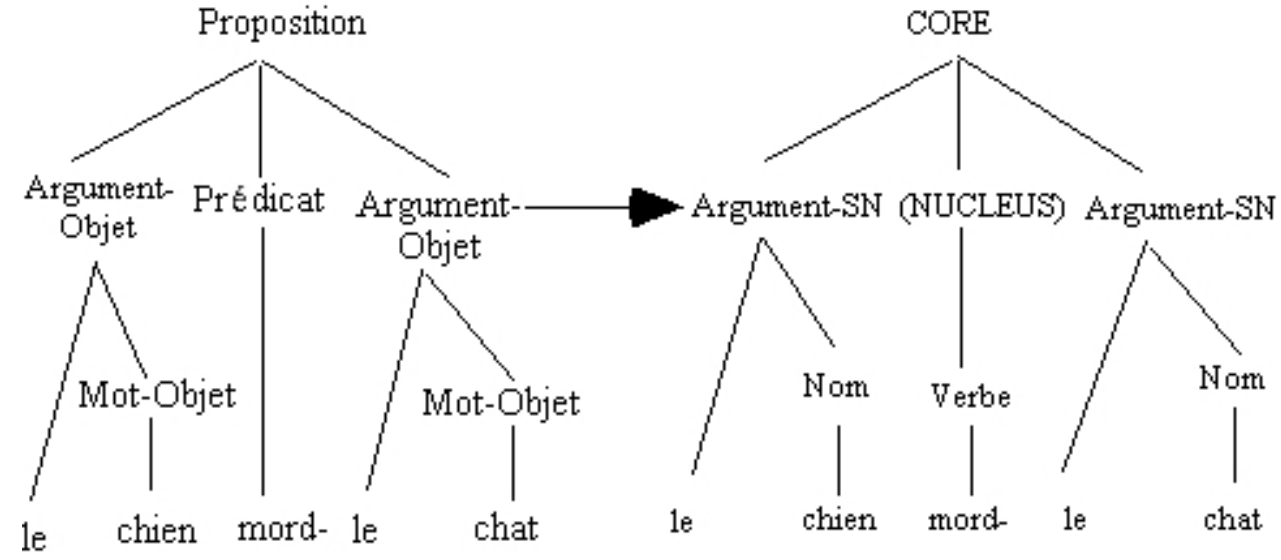

En faveur de la thèse de Braine, il soulignent qu'"il y a des patrons translinguistiques frappants dans l'acquisition des temps, aspects, modalités et de la négation" (p. 641). Par exemple, les distinctions d'aspect apparaîtraient toujours plus tôt que les distinctions de temps. Ils interprètent ces observations comme un argument développemental pour leur stratification de la "projection fonctionnelle" dans laquelle l'aspect est dans la portée de la modalité objective (root modality) laquelle est elle-même dans la portée du temps et de la modalité subjective épistémique (status) et médiative (evidentials) ${ }^{15}$.

Enfin Tomasello (2000) présente une expérience développementale fascinante dans laquelle il a enregistré $10 \%$ de tous les énoncés d'une enfant de 2 ans en interaction avec sa mère durant 6 semaines. Chaque énoncé-cible a été ensuite comparé avec les énoncés 
similaires produits précédemment par l'enfant dans l'enregistrement. Tomasello qualifie de fondés sur l'usage ${ }^{16}$ les opérations syntaxiques réalisées par l'enfant dans les derniers jours de la période, car "l'enfant assemblait ses énoncés à partir d'un assortiment hétéroclite de différents types d'unités psycholinguistiques pré-existantes" (p. 74) par couper-coller de constructions testées et dominées antérieurement.

Tomasello conclut que le matériau stocké, rappelé et réemployé par l'enfant est hautement diversifié, "incluant de tout depuis les mots isolés jusqu'à des catégories abstraites, des énoncés partiellement abstraits ou des schèmes syntaxiques" (p. 77) et que les enfants ne coordonnent pas seulement les formes linguistiques impliquées mais également les fonctions de communications conventionnelles de ces formes, comme le postulent Langacker dans la Cognitive grammar et C. Fillmore, P. Kay et A. Goldberg dans la Construction grammar.

\subsection{Le paradoxe de l'acquisition du langage : réel ou imaginaire?}

La revue Cognitive Linguistics a été le théatre d'une intéressante controverse en quatre actes sur la validité du "paradoxe de l'acquisition du langage" pointé par Jackendoff $(1996,1997)$ et sur la conséquence épistémologique qu'il en tire. Le paradoxe est selon Jackendoff que la communauté linguistique n'ait pas encore réussi à fournir une seule description adéquate de la connaissance qu'un locuteur a de sa propre langue, alors que tout enfant normal arrive à acquérir ce savoir dans un temps limité. Il en conclut que la base de la grammaire universelle est innée.

Hilferty et al. (1998) répliquent que le savoir explicite et le savoir implicite ne doivent pas être confondus et que la grammaire mentale sur laquelle se fonde l'acquisition du langage est un savoir implicite, tandis qu'une grammaire théorique (fondée sur un arsenal de concepts formels) constitue un savoir explicite. Jackendoff (1999) accorde à Hilferty et al. que les grammaires des linguistes et les grammaires mentales sont de nature différente (" two different breeds of cat"). Cependant il considère que d'un point de vue fonctionnel ou informationnel, les distinctions du langage sont les mêmes, qu'elles soient codées explicitement ou implicitement. Il croit comprendre que pour ces auteurs le savoir implicite (de la grammaire mentale) ne pourra jamais être modélisé correctement et il considère que c'est un lourd prix à payer pour "purger l'innéité de l'acquisition du langage". Enfin Meira (2001) reprend le débat en y introduisant l'idée que le choix de l'enfant en phase d'acquisition puisse résulter, non de l'application d'un paramètre clairement identifié, mais d'un jeu complexe et actuellement inanalysable de contraintes variées :

"maybe the child does not "guess" what feature to choose; maybe its cognitive system simply develops in such a way that patterns which seem to reflect the "choice of a parameter" occur spontaneously" (2001: 93) 


\section{La simulation par réseaux neuronaux de l'acquisition du langage et de la transmission des langues}

\subsection{Simuler la manifestation de la faculté de langage (J. Elman} 1999)

Jeffrey Elman, dont j'ai évoqué plus haut l'ouvrage de 1996 sur la révision de la théorie de l'innéité à la lumière de simulations par réseaux neuronaux, présente dans son article de 1999 l'une de ces simulations dont l'objectif est de reconstituer la manière dont un enfant arrive à repérer les régularités syntaxiques dans les énoncés auxquels il/elle est confronté(e) et comment il/elle les réplique.

Elman sélectionne un corps de catégories syntaxiques incluant trois types de verbes: intransitifs, transitifs avec objet obligatoire et transitifs avec objet omissible, et quatre types de relatives avec pronom sujet ou objet et un antécédent qui exerce la fonction de sujet ou d'objet dans la proposition matrice. Il se donne ainsi les moyens de tester la capacité du "comprenant" (comprehender) simulé à identifier dans ces constructions relatives les relations correctes entre $\mathrm{SN}$ et verbes par leur position et leur accord. Trois simulations sont menées successivement.

Dans la première, on attend du comprenant qu'il constitue sa grammaire interne en traitant 10000 paires d'énoncés dotés d'un jugement de grammaticalité. Comme ces énoncés mélangent des phrases simples et complexes, il est incapable de constituer la moindre grammaire.

Dans la seconde, le comprenant est exposé progressivement à des phrases qui comportent une proportion régulièrement croissante de phrases complexes. Dans ce cas, le comprenant parvient bien à constituer une grammaire, mais Elman souligne que c'est une simulation non réaliste du bain linguistique, lequel - en dépit des simplifications syntaxiques qu'entretient le discours de la mère (motherese) - comprend des phrases complexes dès le début.

Le matériel de la troisième simulation adjoint l'introduction d'un filtre attentionnel plausible et confirmé par des observations expérimentales. La capacité attentionnelle du "comprenant" est réglée au début à un niveau bas et est ajustée de manière à augmenter régulièrement au fur et à mesure que de nouveaux énoncés sont traités. Cela signifie que dans un premier temps les phrases complexes sont perçues uniquement comme du bruit et ne sont pas traitées du tout. Par exemple, les phrases traitées peuvent être au début seulement celles qui n'ont aucune proposition relative, puis dans un second temps celles qui ont un pronom sujet, puis enfin celles qui ont un pronom objet. Le résultat est analogue à celui de la seconde simulation, mais dans des conditions "écologiques".

L'intérêt majeur de cette troisième simulation est sa compatibilité avec l'observation que les enfants sont aptes à apprendre leur langue maternelle et des langues étrangères avec une aisance analogue durant un intervalle de temps strictement limité dans lequel le développement de la capacité attentionnelle n'a pas atteint son maximum. Après cette période, c'est la pleine maturation de la capacité attentionnelle qui entrave l'acquisition aisée d'une nouvelle langue. 


\subsection{Simuler la transmission des langues (S. Kirby 2001) ${ }^{17}$}

Simon Kirby poursuit une idée fondamentale : la transmission du langage à travers des générations d'auditeurs-locuteurs et de ce fait l'évolution des langues résultent d'un équilibre entre des pressions opposées sur l'activité de réception et l'activité de production. D'un côté, l'apprenant accorde sa préférence aux énoncés compositionnels et donc transparents. De l'autre, le transmetteur préfère produire des énoncés brefs non compositionnels et donc opaques plutôt que des énoncés compositionnels plus longs et complexes, ce qui exige un effort supplémentaire.

Kirby a monté une série de trois simulations fondées sur la transmission d'une grammaire constituées de 50 paires de deux unités sémantiques $\left\{a^{0}-a^{4}\right\} \&\left\{b^{0}-b^{4}\right\}$ et deux syntagmes respectant un principe de compositionnalité sémantique. Initialement, le système de communication n'est pas structuré, c'est-à-dire qu'il n'y a pas de correspondance établie entre les paires de significations et les paires de syntagmes.

Dans la première simulation, le modèle d'apprentissage itératif (iterated learning model) est réglé de manière à ne prendre en compte que les pressions du côté de l'apprenant, ce qui confère un avantage à la compositionnalité. Au bout d'un certain nombre de générations, un système préservant la structure émerge qui évolue à chaque génération dans ses détails, mais sans permettre aucune irrégularité. Ce résultat est donc un mauvais modèle de la constitution des langues.

Dans la seconde, on ajoute les pressions du côté du transmetteur, ce qui introduit dans le jeu des pressions une certaine priorité à la brièveté. Ainsi entre deux chaînes de différente longueur sémantiquement équivalentes, c'est la plus courte qui est sélectionnée. Par ailleurs l'érosion par des erreurs d'expression est prise en compte. Le résultat est que quelques irrégularités sont produites, mais elles ne persistent qu'un petit nombre de générations au bout desquelles l'apprenant les régularise, ce qui n'est pas conforme à la réalité diachronique.

La troisième simulation part de la constatation que les 10 verbes anglais les plus fréquents : be, have, do, say, make, go, take, come, see et get ont un passé formé de manière irrégulière. Le matériel de la seconde simulation est donc enrichi d'une fonction qui accorde une échelle de fréquence d'usage aux deux significations de sorte que $\mathrm{a} 0>\mathrm{a} 1>\mathrm{a} 2$ $>\mathrm{a} 3>\mathrm{a} 4$ et $\mathrm{b} 0>\mathrm{b} 1>\mathrm{b} 2>\mathrm{b} 3>\mathrm{b} 4$. Le résultat est que les expressions rares sont fortement compositionnelles, tandis que les plus fréquentes sont atomiques, composées d'un morphème $+\emptyset$. Le système est moins stable qu'auparavant, mais les expressions atomiques irrégulières restent inchangées durant des centaines de générations, ce qui est conforme à l'évolution typique des systèmes grammaticaux.

Pour mieux comprendre le processus de constitution et de stabilisation des irrégularités, considérons le détail du paradigme produit par le modèle d'apprentissage itératif sur le tableau 4 qui montre l'état du système à la $127^{\mathrm{e}}$ génération (l'auteur signale que les trois expressions irrégulières $\{\mathrm{g}, \mathrm{y}, \mathrm{s}\}$ restent inchangées jusqu’à la $464^{\mathrm{e}}$ génération).

Tableau 4 : Régularités et irrégularités à la $127^{\circ}$ génération du système de simulation produit par le modèle d'apprentissage itératif de Kirby (2001)

\begin{tabular}{|l|l|l|l|l|l|}
\hline & $a^{0}$ & $a^{1}$ & $a^{2}$ & $a^{3}$ & $a^{4}$ \\
\hline
\end{tabular}




\begin{tabular}{|l|l|l|l|l|l|}
\hline $\mathrm{b}^{0}$ & $\mathbf{g}$ & $\mathrm{s}$ & $\mathrm{k} \cdot \mathrm{f}$ & $\mathrm{j} \cdot \mathrm{f}$ & $\mathrm{uhl} \cdot \mathrm{f}$ \\
\hline $\mathrm{b}^{1}$ & $\mathrm{y}$ & $\mathrm{jg} \cdot \mathrm{i}$ & $\mathrm{k} \cdot \mathrm{i}$ & $\mathrm{j} \cdot \mathrm{i}$ & $\mathrm{uhl} \cdot \mathrm{i}$ \\
\hline $\mathrm{b}^{2}$ & $\mathrm{y} \cdot \mathrm{q}$ & $\mathrm{jg} \cdot \mathrm{q}$ & $\mathrm{k} \cdot \mathrm{q}$ & $\mathrm{j} \cdot \mathrm{q}$ & $\mathrm{uhl} \cdot \mathrm{q}$ \\
\hline $\mathrm{b}^{3}$ & $\mathrm{y} \cdot \mathrm{bq}$ & $\mathrm{jg} \cdot \mathrm{bq}$ & $\mathrm{k} \cdot \mathrm{bq}$ & $\mathrm{j} \cdot \mathrm{bq}$ & $\mathrm{uhl} \cdot \mathrm{bq}$ \\
\hline $\mathrm{b}^{4}$ & $\mathrm{y} \cdot$ upeg & $j g \cdot$ upeg & $\mathrm{k} \cdot \mathrm{upeg}$ & $\mathrm{j} \cdot$ upeg & uhl $\cdot$ upeg \\
\hline
\end{tabular}

En faisant provisoirement abstraction des trois résultats irréguliers, à savoir :

\begin{tabular}{|l|l|}
\hline expression & contenu \\
\hline $\mathbf{g}$ & $\{\mathrm{a} 0, \mathrm{~b} 0\}$ \\
$\mathbf{y}$ & $\{\mathrm{a} 0, \mathrm{~b} 1\}$ \\
$\mathbf{s}$ & $\{\mathrm{a} 1, \mathrm{~b} 0\}$ \\
\hline
\end{tabular}

nous pouvons vérifier que chaque syntagme est composé de deux parties. J'ai ajouté un $(\bullet)$ entre ces deux parties pour mettre en évidence les régularités dans la composition de ces syntagmes.

Dans chaque paire de significations constituée de $a 0$ et d'une variante de ' $b$ ' à l'exception de 'b0', la signification a0 est exprimé par le constituant 'y'. Comme la paire $\{a 0, b 1\}$ est exprimée par le seul 'y', nous pouvons supposer que le contenu b1 est exprimé irrégulièrement par $\varnothing$ quand il est combiné avec la signification a0. Dans les autres colonnes, nous voyons que la signification a1 est toujours exprimée par la chaîne 'jg', a2 par le constituant ' $k$ ', a3 par 'j' et a4 par la chaîne 'uhl'. Quant aux différentes variantes de la signification $\{b\}$, à l'exception des deux premières paires, bo est exprimée par le constituant 'f', b1 par 'i', b2 par 'q', b3 par la chaîne 'bq' et b4 par la chaîne 'upeg'.

Concernant les deux paires de significations restantes dont l'expression est irrégulière $\{a 0, b 0\}$ et $\{a 1, b 0\}$ il apparaît que le constituant 's' ne fait partie d'aucun autre syntagme, tandis que ' $g$ ' est un constituant régulier de la chaîne 'jg' exprimant la signification a1 et également un constituant régulier de la chaîne 'upeg' exprimant la signification b4. Les trois irrégularités ont donc des sources différentes : 'y' comme expression de la paire de significations $\{a 0, b 1\}$ est un syntagme semi-régulier, c'est-à-dire régulier pour 'y' comme moyen d'exprimer a0 et irrégulier pour $\varnothing$ comme moyen d'exprimer b1. Les deux autres expressions 'g' et 's' sont complètement irrégulieres, 's' parce qu'elle n'apparaît nulle part ailleurs dans le paradigme et ' $g$ ' parce qu'elle se présente comme un constituant de chaînes gauches et droites à différentes places dans le paradigme.

Pour quelle raison la paire de significations $\{a 4, b 4\}$ est-elle exprimée par le syntagme le plus long : 'uhl•upeg' constitué d'une chaîne gauche de trois constituants et d'une chaîne droite de quatre? Comme nous l'avons vu, le système simule des pressions de deux origines: une pression favorisant la compositionalité du côté de l'apprenant et une pression en faveur de la brièveté du côté du transmetteur. Les deux types de pressions comportent des désavantages : les expressions compositionnelles sont longues et sont une cause d'erreur pour le locuteur, tandis que les expressions brèves et non compositionnelles sont opaques (cf. Tableau 5). 
Tableau 5 : Avantage et inconvénient attaché au mode d'expression favorisé respectivement par l'apprenant et le transmetteur

\begin{tabular}{|l|l|l|}
\hline & $\begin{array}{l}\text { expression compositionnelle } \\
\text { favorisée par l'apprenant }\end{array}$ & $\begin{array}{l}\text { expression non compositionnelle } \\
\text { favorisée par le transmetteur }\end{array}$ \\
\hline avantage & transparence & brièveté \\
\hline inconvénient & longueur & opacité \\
\hline
\end{tabular}

Mais le facteur supplémentaire de la fréquence d'usage adjoint au modèle d'apprentissage sélectionne les syntagmes destinés à être brefs et opaques - ici ceux qui expriment les paires de significations les plus fréquentes : $\{a 0, b 0\},\{a 0, b 1\}$ et $\{a 1, b 0\}-$ et ceux qui seront compositionnels et donc plus longs.

Imaginons maintenant une application du modèle d'apprentissage pour un paradigme de flexion verbale. Suivant l'exemple fourni pas Kirby de la flexion des verbes les plus fréquents de l'anglais, les significations a0-a4 peuvent être conçues comme étant des classes de significations de lexèmes verbaux avec une fréquence d'usage décroissante et les significations bo-b4 comme des combinaisons de propriétés flexionnelles concernant par exemple les catégories du temps (actuel vs. inactuel) et du mode (déclaratif vs. distancié).

61 Pour les besoins de la démonstration, nous supposerons par simplification que les valeurs sémantiques \{actuel, inactuel, déclaratif, distancié\} sont respectivement exprimées par les catégories paradigmatiques \{présent, imparfait, indicatif, subjonctif\}. Supposons que bo combine par exemple \{déclaratif, actuel\} et b4 \{distancié, inactuel). L'ordre de b0 à b4 suit une fréquence décroissante. Pour les paires de significations lexicale et flexionnelle hautement récurrentes, des irrégularités sont attendues, parce que le besoin de brièveté l'emporte sur le besoin de transparence, donc de compositionalité. On peut l'illustrer à l'aide des verbes être et avoir de fréquence maximale en raison, pour être, de son triple statut d'auxiliaire de temps, de voix et de copule, et pour avoir de son double statut d'auxiliaire de temps/aspect et de verbe plein et en leur adjoignant le verbe faire qui a également une haute fréquence en tant qu'auxiliaire de la voix causative et que proforme de tous les verbes d'actions (ex. je ne fais rien d'autre que de $\mathrm{INF}_{\text {<action }}$ ).

Sur le tableau 6, on voit qu'aucun des trois verbes ne présente d'irrégularité à l'imparfait du subjonctif (censé exprimer la distanciation et l'inactualité), alors que être présente 6 formes irrégulières sur six, avoir quatre et faire deux à l'indicatif présent (censé exprimer le mode déclaratif et l'actualité). Le fragment de paradigme de conjugaison de ces trois verbes fournit ainsi une illustration de la validité de l'hypothèse de base de la troisième simulation qui est que l'apprenant exerce une pression en faveur de la transparence, le transmetteur une pression comparable en faveur de la brieveté et que le poids respectif de ces deux pressions est fonction de la fréquence d'usage : lorsque l'une et/ou l'autre des deux significations est rare, leur expression l'est aussi et la pression en faveur de la transparence l'emporte sur la pression en faveur de la brièveté, en revanche lorsque les deux significations sont fréquentes, c'est l'inverse qui se produit et les irrégularités (formes brèves et opaques) s'installent et se stabilisent pour de nombreuses générations. 
Tableau 6 : Illustration de l'effet de la fréquence sur les irrégularités flexionnelles (originalilté de la $3^{\mathrm{e}}$ simulation de Kirby 2001)

\begin{tabular}{|c|c|c|c|}
\hline & $\begin{array}{l}\text { bo : } \\
\text { \{déclaratif \& actuel\} } \\
\text { Æ indicatif présent }\end{array}$ & $(\mathrm{b} 1, \mathrm{~b} 2, \mathrm{~b} 3)$ & $\begin{array}{l}\text { b4 : } \\
\{\text { distancié \& inactuel }\} \\
\text {-> subjonctif imparfait }\end{array}$ \\
\hline $\begin{array}{l}\text { a0 : } \\
\text { être }\end{array}$ & $\begin{array}{l}\text { suis, es, est, } \\
\text { sommes, êtes, sont } \\
\text { [6 irrégularités] }\end{array}$ & $(\ldots)$ & $\begin{array}{l}\text { fusse, fusses, fût, } \\
\text { fussions, fussiez, fussent } \\
\text { [Ø irrégularité] }\end{array}$ \\
\hline $\begin{array}{l}\text { a1: } \\
\text { avoir }\end{array}$ & $\begin{array}{l}a i, a s, a, \\
\text { avons, avez, ont } \\
\text { [4 irrégularités] }\end{array}$ & $(\ldots)$ & $\begin{array}{l}\text { eusse, eusses, eût, } \\
\text { eussions, eussiez, eussent } \\
\text { [Ø irrégularité] }\end{array}$ \\
\hline $\begin{array}{l}\text { a2: } \\
\text { faire }\end{array}$ & $\begin{array}{l}\text { fais, fais, fait, } \\
\text { faisons, faites, font } \\
\text { [2 irrégularités] }\end{array}$ & $(\ldots)$ & $\begin{array}{l}\text { fisse, fisses, fît, } \\
\text { fissions, fissiez, fissent } \\
\text { [Ø irrégularité] }\end{array}$ \\
\hline
\end{tabular}

Les méthodes de simulation d'Elman et de Kirby se révèlent donc manifestement apparentées et leurs objectifs sont compatibles: Elman montre "l'importance de commencer petit", c'est-à-dire de tenir compte du développement des capacités attentionnelles de l'apprenant et de choisir le bon intervalle de temps pour lancer l'acquisition. Kirby montre au surplus que dans le processus de réplication et de transmission le besoin d'économie du point de vue du producteur est aussi significatif que le besoin de transparence - ou de compositionalité, c'est-à-dire d'iconicité entre la composition sémantique et la composition morphosyntaxique $-\mathrm{du}$ point de vue de l'apprenant et que le poids respectif des deux pressions est fonction de la fréquence d'usage.

\section{Bilan}

Aucun des linguistes, psycholinguistes et "infolinguistes" évoqués ici ne se rattache à la thèse de ce que Croft (1995) appelle le fonctionalisme "extrême", celui d'une grammaire en émergence permanente à partir du discours (thèse de Paul Hopper, 1987, sévèrement critiquée au sein même du fonctionalisme par Givón, 1995a et 1999). Mais, à l'exception de S. Anderson et F. Newmeyer, tous rejettent la conception de la faculté de langage développée par les chercheurs du M.I.T., celle d'un module du langage suivant des principes spécifiques par rapport aux autres composantes de l'activité cognitive (Fodor 1983) et plus récemment celle du langage comme instinct (Pinker 1994).

Ce que la plupart des travaux mentionnés ici cherchent à démontrer, c'est que l'internalisation des régularités grammaticales est indissociable de l'identification des contenus et des intentions de communication (Goldberg ${ }^{18}$ ), que les principes innés qui gouvernent la constitution de la grammaire mentale sont fondamentalement d'ordre sémantique (Braine, van Valin \& LaPolla), que l'apprentissage de la production discursive fonctionne par récupération de fragments de discours associés à une situation 
(Tomasello) et que les réseaux neuronaux constituent un outil exceptionnel pour modéliser le mode de transmission des paires \{expression \& contenu\} linguistiques par stabilisation d'un jeu de motivations opposées (Elman, Kirby).

\section{BIBLIOGRAPHIE}

ALLEN, J. \& SEINDENBERG, M. 1999. "The emergence of grammaticality in connectionist networks" in B. Mac Whinney (ed.), 115-15

ANDERSON, S. 1999. "A formalist's reading of some functionalist work in syntax". In Darnell et al. (eds), vol. I, 111-135

BALDWIN, J.-M. 1902. Development and Evolution. New York: Macmillan.

BRAINE, M. 1992. "What sort of innate structure is needed to 'bootstrap' into syntax?", Cognition $45,77-100$.

CROFT, W. 1993. "Functional-typological theory in its historical and intellectual context", Sprachtypologie und Universalienforschung 46. 15-26

CROFT, W. 1995. "Autonomy and functionalist linguistics", Language 71. 490-532.

CROFT, W., 1998a. "La théorie de la typologie fonctionnelle dans son contexte historique et intellectuel", [traduction de la version révisée et mise à jour de Croft (1993)], Verbum 1998/3 : 289-307.

CROFT, W. 1998b. "The structure of events and the structure of language", in M. Tomasello (ed.), 67-92

CROFT, W. 2000. Explaining Language Change. An Evolutionary Approach. Harlow, Longman.

DARNELL, M. et al.1999. Functionalism and Formalism in Linguistics [2 volumes]. Amsterdam, Benjamins

DEACON, T. 1997. The Symbolic Species - The Co-evolution of Language and the Brain. New-York: Norton.

DOWTY, D. 1979. Word Meaning and Montague Grammar. Dordrecht : Reidel

DUBoIS, J. 1985. "Competing motivations" in J. Haiman ed., Iconicity in syntax. Amsterdam :

Benjamins. 343-365.

ELMAN, J. et al. 1996. Rethinking Innateness, A Connectionis Perspective on Development. Bradford Book, MIT Press

ELMAN, J. 1999. "The emergence of language: a conspiracy theory". in B. Mac Whinney (ed.), The emergence of Language. Mahwah, New-Jersey: Erlbaum, 1-27

FODOR, J. 1983. The Modularity of Mind. Cambridge (Mass.): M.I.T. Press.

FRANÇOIS, J. 1998. "Grammaire fonctionnelle et dynamique des langues : de nouveaux modèles d'inspiration cognitive et biologique". Verbum XX/3 : 223-256. Presses Universitaires de Nancy. 
FRANÇoIS, J. 2001. Compte-rendu de Hansjakob SEILER, Linguistic Universals Research : A Synthesis. Cahiers Ferdinand de Saussure 54, 503-8.

FRANÇOIS, J. (à paraître) Recent advances in linguistic functionalism, Conférence invitée au Congrès de la Societas Linguistica Europaea, Louvain, août 2001 ; à paraître dans N. Delbecque et al. (eds.), Linguistic currents and conceptions. Louvain: Peeters.

GIVÓN, T. 1995a. Functionalism and Grammar. Amsterdam : Benjamins.

GIVóN, T. 1995b. "Coherence in text vs. coherence in mind", in M. A. Gernsbacher \& T. Givón eds. 1995. Coherence in Spontaneous Text. Amsterdam : Benjamins. 59-115.

GIVÓN, T. 1998a. "On the co-evolution of language, mind and brain", Evolution of Communication 2-1: 45-116.

GIVÓN, T. 1998b. "The functional approach to grammar" in Tomasello (ed).

GIVÓN, T., 1998c. "L’approche fonctionnelle de la grammaire" Verbum 1998/3 : 257-288

GIVÓN, T. 1999. "Generativity and variation: The notion 'rule of grammar' revisited" in Mac Whinney (ed.), 81-114 (Chapter 3).

GOLDBERG, A. 1998. "Patterns of experience in patterns of language" in M. Tomasello (ed.), 203-219 GOLDBERG, A. 1999. "The emergence of the semantics of argument structure constructions" in W. McWhinney (ed.) Chapter $7: 197-212$

HAGÈGE, C. 1998. "Grammaire et cognition. Pour une participation de la linguistique des langues aux recherches cognitives", Bulletin de la Société de Linguistique de Paris, XCIII, fasc.1 : 41-58. HEINE, B. 1997a. Possession. Cognitives sources, Forces, and Grammaticalization. Cambridge University Press.

HEINE, B. 1997b. Cognitive Foundations of Grammar. Oxford University Press.

HEINE, B. 1997c. "Grammaticalization and language universals", Mémoires de la Société de Linguistique de Paris Tome V. Paris : Klincksiek, 11-23.

hilferty, J., J. VAlenzuala \& O. Villaroya, 1998. "Paradox lost", Cognitive Linguistics 9, 175-180.

HOPPER, P. J. 1987. "Emergent grammar", in Grammar and Cognition, 13th Annual meeting of the Berkeley Liguistic Society. Fels 1987. Berkeley. 139-157.

HURFORD, J. 1999. "Functional innateness. Explaining the critical period for language acquisition" in Darnell et al. (ed.), Volume II, 341-363.

JACKENDOFF, R. 1996. "Conceptual semantics and cognitive linguistics", Cognitive linguistics 7, 93-129.

JACKENDOFF, R. 1997. The Architecture of the Language Faculty. Cambridge (Mass.): MIT Press.

JACKENDOFF, R. 1999. "Paradox regained ", Cognitive Linguistics 10: 271-277.

KINTSCH, W. 1995). "How readers construct situation models for stories: The role of syntactic cues and causal inferences", in M.A. Gernsbacher \& T. Givón (eds., 1995). Coherence in Spontaneous Text. Amsterdam : Benjamins, 139-160

KIRBY, S. 1997. "Competing motivations and emergence: Explaining implicational hierarchies", Linguistic Typology, 1997/1, 5-31. 
KIRBY, S. 1998. "Motivations concurrentes et émergence comme explications des hiérarchies implicationnelles", Verbum XX-3, 309-336. [traduction de Kirby 1997]

KIRBY, S. 1999. Function, Selection, and Innateness - The Emergence of Language Universals. Oxford: Oxford University Press.

KIRBY, S. 2001. "Spontaneous Evolution of Linguistic Structure - An iterated learning model of the emergence of regularity and irregularity", IEEE Transactions on Evolutionary Computation. 5.2: 102-110.

LAMB, S. 1999. Pathways of the Brain. The Neurocognitve Basis of Language. Amsterdam: Benjamins.

LANGACKER, R. 1995. "Structural syntax: The view from Cognitive Grammar", in F. Madray-Lesigne \& J. Richard-Zappella (eds. 1995). Lucien Tesnière aujourd'hui. Louvain: Peeters. 13-39

LASS, R. 1980. On Explaining Language Change. Cambridge: Cambridge University Press.

LINEBARGER, M. C., SCHWARTZ, M. F. \& SAFFRAN, E. M. 1983. "Sensitivity to grammatical structure in so-called agrammatic aphasics", Cognition 13: 361-392.

MAC WHINNEY, B. (ed.) 1999. The Emergence of Language. Mahwah, New-Jersey

MEIRA, S. 2001. "Paradox escaped?", Cognitive Linguistics 12-1, 89-96

NETTLE, D. 1999. "Functionalism and its difficulties in biology and linguistics", in Darnell et al. (eds) volume I: 445-467.

NEWMEYER, F. 1998. Language Form and Language Function. Cambridge (Mass.): M.I.T. Press.

NEWMEYER, F. 1999. "Some remarks on the functionalist-formalist controversy in linguistics", in Darnell et al. (eds.) volume I: 469-486.

PiATtelli-PAlmerini, M. (ed.) 1979. Théories du langage - Théories de l'apprentissage. Le débat entre Jean Piaget et Noam Chomsky. Paris : Le Seuil.

PINKER, S. 1994. The Language Instinct: How the Mind Creates Language. New-York: William Morrow. RICHARDS, R. 1987. Darwin and the Emergence of Evolutionary Theories of Mind. Chicago: University of Chicago Press.

SEILER, H. 2001. Language Universals Research: A Synthesis. Tübingen : Gunter Narr.

TOMASELlo, M. (ed.) 1998. The New Psychology of Language - Cognitive and Functional Approaches to Language Structure. Mahwah, NJ: LEA.

TOMASELlo, M. 2000. "First steps toward a usage-based theory of language acquisition", Cognitive linguistics 11-1/2, 61-82.

VAN VALIN Jr., R. 1996. "Role and Reference Grammar", in K. Brown \& J. Miller (eds.) The Concise Encyclopedia of Syntactic Theories. Oxford: Pergamon Press, 281-294.

VAN VALIN Jr., R. 2001. An introduction to Syntax. Cambridge: Cambridge University Press.

VAN VALIN Jr., R. \& LAPOLLA, R. 1997. Syntax: Structure, Meaning, Function, 640-9 


\section{NOTES}

1. Extrait de l'entretien entre H. Seiler et J. François (Lenzburg, 31 mars / $1^{\mathrm{er}}$ avril 2001) à paraître en traduction française dans la "Biobibliographie de Hansjakob Seiler " éditée par P. Swiggers \& J. François chez Peeters.

2. Outre l'édition du numéro 1998/3 de VERBUM mentionné plus bas, il s'agit d'une conférence à l'université de Caen le 27 mai 1999 consacrée à L'inspiration cognitive et biologique dans le renouvellement actuel de la linguistique fonctionnelle, de ma conférence à la journée scientifique "Les linguistiques cognitives, Etat des lieux et perspectives" de l'Action Concertée Incitative Cognitique (CNRS, ENS Ulm, 1er décembre 2000), intitulée Les orientations cognitives du fonctionnalisme et de ma conférence invitée au congrès annuel de la Societas Linguistica Europaea à Louvain (30 août 2001) intitulée Recent advances in functional linguistics (à paraître dans les Actes du congrès).

3. Ce modèle est largement développé dans Croft (2000).

4. Le premier volume, consacré à la confrontation des positions par thèmes, constitue les actes d'un symposium contradictoire du même type que celui qui avait opposé Chomsky et Piaget en 1975 sur les modèles d'acquisition du langage (cf. Piattelli-Palmerini 1979). Le second est constitué de contributions par théorie et par domaine d'application.

5. Sur la théorie des universaux de Hansjakob Seiler et la priorité méthodologique que ce dernier donne à la recherche sur les universaux par rappport à la typologie des langues contrairement à l'esprit du temps, je renvoie à François (2001) et François (à paraître)

6. En ce qui concerne les théories évoquées dans la section 3 et les méthodes de simulation neuronale apppliquées dans la section 5, je n'ai évidemment pas compétence pour les évaluer et je conçois mon rôle comme celui d'un médiateur attirant l'attention sur des travaux bien argumentés et empiriquement valides.

7. Le modèle de Role and Reference Grammar développé par R. van Valin Jr. comporte deux formalismes syntaxique et sémantique corrélés par une fonction de linking. L'articulation entre syntaxe et sémantique exclut d'en faire un modèle strictement computationnel.

8. Au sens de standard téléphonique, c'est-à-dire de poste de distribution.

9. Je présente en italiques les citations dans la langue originale et en caractères romains les citations traduites.

10. Il est à noter que l'argumentation de Goldberg $(1998,1999)$ s'applique particulièrement bien à l'anglais en raison de la souplesse extrême de cette langue pour insérer un verbe dans une construction a priori incompatible. Le français ne se prête pas à une telle gymnastique (les stylistiques comparées français / anglais et français / allemand l'ont largement illustré depuis longtemps), raison pour laquelle je conserve les illustrations en anglais.

11. Il s'agit de 'light verbs' qui sont acquis en premier.

12. La construction transitive élémentaire N1 V N2 peut accueillir par compactage lexical sur le verbe ou par conversion $\mathrm{X}>\mathrm{V}$ une variété de constituants actanciels ou circonstanciels plus ou moins étendue selon les langues. Alors que le français ne peut accueillir dans le matériau morphologique du verbe que des actants, par exemple le directionnel (N1 conduit N2 à l'hôpital > $\mathrm{N} 1$ hospitalise N2) et l'instrument (ex. N1 coupe N2 avec une scie > N1 scie N2), l'anglais étend cette liberté à d'autres types de circonstants (ex. N1 sends N2 to N3 over night > N1 overnights N2 to N3).

13. Cf. mon résumé de l'argumentation de Givón (1995a: Chapitre IX) dans François (1998: 245-6). Givón (1998: 105) résume sa thèse ainsi: "the rise of human communication is, I suggest, a paradigm case of co-evolution, a multi-causal interaction of cultural, behavioral, communicative and neuro-cognitive factors."

14. Cet argument vaut sans doute contre une conception des universaux substantiels (par exemple des parties du discours) mais il n'a pas accès aux régularités plus profondes des 
universaux implicationnels de J. Greenberg et encore moins aux universaux très abstraits de structuration des "dimensions" de l'Appréhension, de la Participation ou de l'Identification selon Seiler (2001).

15. Cette stratification pose le problème épineux de la délimitation de la catégorie de l'aspect : si pour le français on entend par "aspect" la catégorie de la phase (cf. commencer à, s'arrêter de, finir de), l'aspect est bien dans la portée de la modalité objective (ex. Marie doit commencer à préparer ses valises), mais si on entend par là la visée aspectuelle (venir de, être sur le point de), c'est l'inverse (ex. Marie vient de / est sur le point de pouvoir obtenir son visa de séjour). Si une périphrase aspectuelle est dans la portée d'un verbe de modalité, celui-ci exprime une modalité sujective, ex. Marie peut être en train de boucler ses valises $\approx$ Peut-être Marie est-elle en train de boucler ses valises.

16. Tomasello désigne comme "fondées sur l'usage" un ensemble de théories du langage incluant les travaux de grammaire fonctionnelle récente et de grammaire cognitive (cf. note 1).

17. L'article de S. Kirby présenté ici s'inscrit dans une succession de travaux, dont un article de 1997 traduit dans le numéro de Verbum 1998/3 et introduit extensivement dans François (1998 : 248-251) et sa thèse publiée en 1999.

18. Voir aussi le modèle de "linguistique neurocognitive" élaboré récemment par S. Lamb (1999), où l'auteur montre la variabilité du mode de corrélation entre un contenu conceptuel et une expression linguistique d'un locuteur à un autre et en fonction du contexte d'énonciation.

\section{RÉSUMÉS}

La spécificité de la faculté de langage est l'objet d'une controverse fondamentale entre les représentants de la modularité de l'esprit et spécifiquement d'une grammaire universelle modulaire concernée seulement par la modélisation de la compétence linguistique communément désignés comme "formalistes" - et les défenseurs d'une vision fonctionaliste dont la dominante peut être pragmatique ou cognitive. Le propos de cet article est en premier lieu d'éclairer le débat épistémologique entre fonctionalisme et formalisme en linguistique. Puis, après avoir évoqué brièvement la corrélation entre les universaux cognitifs et les universaux du langage, je présenterai les arguments de chercheurs d'orientation fonctionaliste sur l'innéité des propriétés du langage et sur le 'paradoxe' de l'acquisition des structures linguistiques selon Jackendoff, avant d'évoquer deux tentatives de simulation par réseaux neuronaux de l'ontogénèse du langage et de la transmission des langues. La première concerne la constitution et la stabilisation de la syntaxe de la transitivité et de la relativisation, la seconde illustre la nécessité de prendre en compte les exigences communicationnelles du locuteur et celles de l'allocutaire pour simuler la transmission efficace de signes linguistiques entre deux générations de locuteurs.

The specifity of the faculty of language has been the scope of a fundamental controversy between the defenders of mind modularity and more specifically of a modular universal grammar only concerned with modelling the linguistic competence commonly known as formalists and the defenders of a functionalist view whose prevailing option may be either pragmatic or cognitive. The purpose of this paper is first and foremost to shed light on the epistemological debate between functionalism and formalism in linguistics. In continuation, after briefly reviewing the correlation between cognitive and linguistic universals, I will present the arguments of functionalist researchers about the innate character of the properties of language and about the 
paradox of the acquisition of linguistic structures in Jackendoff's model, before presenting two attempts at simulating the ontogenesis of language and language transmission by neural networks.The former deals with the constitution and stabilization of the syntax of transitivity and of relativization, and the latter illustrates the necessity to take into account the speaker's and hearer's communicational requirements to simulate the efficaciousness of the transmission of linguistic signs between two generations of speakers.

INDEX

Mots-clés : acquisition, cognitive, fonctionnelle, grammaire, langage, sémantique

\section{AUTEUR}

JACQUES FRANÇOIS

Université de Caen - CRISCO (UMR 6170) 